\title{
IS EVERY GO-TOPOLOGY A JOIN OF TWO ORDERABLE TOPOLOGIES?
}

\author{
PAUL R. MEYER, VICTOR NEUMANN-LARA AND RICHARD G. WILSON ${ }^{1,2}$
}

\begin{abstract}
As a generalization of GO-topologies ( $\mathrm{GO}=$ generalized ordered), we are interested in those topologies (here called JO-topologies) on a set $X$ which can be expressed as a join of orderable topologies (the join being taken in the lattice of all topologies on $X$ ). If a topology $t$ is the join of $m$ orderable topologies we say $t$ is $\mathrm{JO}[\mathrm{m}]$. It is not difficult to prove that every GO-topology is a JO-topology, but the question (raised in [M 71]) as to whether every GO-topology is JO[2] seems much more difficult). We show that $X$ is $\mathrm{JO}[2]$ if $X$ is a subspace of an orderable space $D$, where $D$ is either metrizable and locally separable, or connected with countable cellularity. (The theorem is actually more general than is stated here.) We give an example to show that for any positive integer $n$ there is a finite join of order topologies which is not JO[n], but these are not GO-topologies.
\end{abstract}

1. Background and preliminary results. The notion of generalized ordered space was introduced by Čech in the 1930s (see [C, p. 286]). Since then they have been studied from various points of view by many authors (see for example [L 71, F, P 77, P 81]; see Lutzer [L 80] for a recent survey with further references). Such spaces have also been called suborderable [P 77]; we prefer the latter term as being more descriptive (GO-spaces are those spaces which can arise as subspaces of orderable spaces), but it does not seem to be widely used. A study of the properties of cardinal functions for the class of finite joins of orderable topologies can be found in [WM].

Let $(X, t,<)$ be a GO-space (notation as in [L 71]); i.e., let $(D,<)$ be linearly ordered topological space (LOTS) containing $X$ as a subspace, with $t=$ relative topology on $X$. A point $x$ in $X$ is called a bad point of $X$ if the $t$-neighborhoods of $X$ are not the same as the induced order neighborhoods; let $B$ denote the set of all bad points. We associate with each bad point in $X$ one or two points in $D-X$ (called missing points), chosen as follows: if $p$ is a bad point from below, then $p$ is the $X$-sup of $\{y \in X: y<p\}$ and $p$ is not the $D$-sup of this set; i.e., there is a point $m$ in $D-X$ such that $\{y \in X: y<p\}<m<p$. The construction for points which are bad from above is analogous. For each bad point choose a missing point below and/or above, and let $M$ denote the set of missing points so chosen.

Received by the editors January 17, 1981 and, in revised form, April 10, 1981.

1980 Mathematics Subject Classification. Primary 54F05; Secondary 54A25, 54A10, 06B30.

Key words and phrases. Orderable topology (LOTS), generalized order topology (GO-topology, suborderable topology), lattice of topologies, join of topologies.

${ }^{1}$ The first and third authors gratefully acknowledge the support of the Consejo Nacional de Ciencia $y$ Tecnologia (Mexico), grant PCCBNAL 790179 and the Consiglio Nazionale delle Ricerche (Italy).

${ }^{2}$ The authors wish to thank the referee for much valuable help in reformulating the proof of the main theorem in a more coherent form. 
THEOREM. If $(X, t,<)$ is any subordered space, then $t$ is a join of $m$ order topologies, where $m=\max \{|M|, 2\}$.

Proof. We may assume that $D=X \cup M$ (see [L 71] and [C, p. 286]). In this case we may use the following notation: each missing point can be written as $b^{+}$or $b^{-}$for some $b$ in $B$. Let $t_{0}$ denote the <-order topology on $X$. For each missing point $p$ we define a topology $t_{p}$ on $X$. If $p=b^{-}$define $t_{p}$ by reversing the $<-$ order on $\{y: y<p\}$. Then $t_{0} \vee t_{p}$ eliminates convergence of nets which $t_{0}$-converge to $p$ from below and agrees with $t_{0}$ otherwise. If $p=b^{+}$modify the above accordingly. Thus $t=t_{0} \vee\left(\bigvee\left\{t_{p}: p \in M\right\}\right)$. If $|M| \geqslant 2$ then $t_{0}$ is not needed.

The following example justifies the assertion in the introduction that finite joins of orderable topologies are not necessarily $\mathrm{JO}[2]$. The modification needed to do this for $\mathrm{JO}[n]$ will be clear. This method was used in [M 71, Example 4] to construct a JO[2] example which was not a GO-space, in fact not even a chain net space; the argument used there also shows that the present example is not GO.

EXAMPLE. A JO[5] topology which is not $\mathrm{JO}[2]$.

Let $X=\left(\omega_{0} \times \cdots \times \omega_{4}\right) \cup\{p\}$, where $p=\left(\omega_{0}, \ldots, \omega_{4}\right)$. For $0<i<4$, let $t_{i}$ be an order topology on $X$ in which $p$ is the last point and the rest of the ordering is defined by that lexicographic ordering on $\Pi\left\{\omega_{j}: 0 \leqslant j \leqslant 4\right\}$ in which the $i$ th coordinate dominates (i.e., $\left(x_{0}, \ldots, x_{4}\right)<\left(y_{0}, \ldots, y_{4}\right)$ means $x_{i}<y_{i}$, or $x_{i}=y_{i}$ and...). (There is a $t_{i}$-neighborhood base at $p$ in which each basic neighborhood is a union of hyperplanes normal to the $i$ th axis and indexed by a tail of $\omega_{i}$. Thus the $t_{i}$-ordering within the hyperplanes need not concern us.) Let $t=t_{0} \vee \cdots \vee t_{4}$. Thus $(X, t)$ is $\mathrm{JO}[5]$ by definition. Assume it is $\mathrm{JO}[2]$ and get a contradiction as follows. It is known [WM] that nets directed by $2 n$-fold products of ordinals suffice to describe the convergence in any $\mathrm{JO}[n]$ topology. Thus there is a net in $X-\{p\}$ directed by a 4-fold product of ordinals converging to $p$. On the other hand the $t$-neighborhoods of $p$ are precisely the relative product neighborhoods in $X$ from $\Pi\left\{\omega_{j}+1: 0 \leqslant j \leqslant 4\right\}$. Some $\omega_{j}$ will not be represented in the 4-fold product, and the hypothesized net will not be cofinal in the $\omega_{j}$ dimension, which is a contradiction.

2. Is every GO-topology $\mathrm{JO}[2]$ ? It is easy to show that the answer is yes for all GO-spaces $X$ in which the set of missing points $M$ is discrete in $D$. It is also known that the answer is yes for the Sorgenfrey line [M 73]. For the Michael line we can show that it is JO[3]. These two lines are perhaps the best known examples of pathological GO-spaces; see [L 71] for background and further properties.

There are of course conditions under which one topology suffices, i.e., the GO-topology is orderable. If a GO-topology is either compact or connected, then it is orderable; in fact the given ordering works [L 71, 6.1]. If a GO-topology is scattered (= dispersed) then it is orderable, but in general not under the given ordering [P 81].

We turn now to our main result, which shows that the answer is yes for a large class of GO-topologies. Since the precise statement of the theorem requires some new terminology, we begin by mentioning some specific cases which are easier to describe. 
COROllary. Let D be a LOTS satisfying any one of the listed conditions and let $X$ be any subspace of $D$. Then $X$ is JO[2]; i.e., the topology can be expressed as a join of two orderable topologies on $X$.

(i) $D$ has countably many jumps and countable cellularity; more generally, $D$ is a disjoint topological sum of such spaces.

(ii) $D$ is metrizable and locally separable.

(iii) $D$ has countable weight.

Note that (iii) is a special case of both (ii) and (i).

EXAMPLE. The product of $\omega$ copies of a discrete space of power $\omega_{1}$ is a metrizable LOTS which is nowhere locally separable. This shows that the second hypothesis in (ii) does not follow from the rest of the hypotheses. The fact that this space is orderable follows from [H].

A few more definitions are needed in order to state the theorem. We define an equivalence relation on a GO-space $X: x \sim y$ means there is no missing point between $x$ and $y$. Let $\mathcal{Q}$ denote the set of all equivalence classes and $\theta$ denote the set of those equivalence classes which contain at least two points.

TheOREM. Let $D$ be a LOTS and $X$ be any subspace of $D$. If the set of missing points $M$ and the set of nontrivial equivalence classes are both countable, then the relative topology on $X$ is JO[2], i.e., it is the join of two orderable topologies.

Proof of Corollary. (i) follows from the Theorem because each missing point and its associated bad point determine in $D$ a jump or an interval. By a result of DeMarco [DM, p. 550] every locally separable metric space is a disjoint topological sum of second countable spaces, whence (ii).

3. Proof of the main result. $(D,<)$ will denote the LOTS in which $X$ is embedded; $M^{+}$(resp. $M^{-}$) will denote the set of all elements of $M$ of the form $b^{+}$ (resp. $b^{-}$) for some $b \in B$. If $S=(a, b)$ is an open interval in $D$ then we denote the element $a$ by $\lambda(S)$ and the element $b$ by $\rho(S)$ (we do not exclude the cases $\lambda(S)=-\infty$ and $\rho(S)=+\infty)$.

Since the sets $M$ and $\theta$ are both countable, we can order them as sequences, say $\left(m_{n}\right)$ and $\left(\pi_{n}\right)$ respectively. We will define by induction a sequence of open intervals $\left(T_{n}\right)$ and two sequences $\left(E_{n}\right)$ and $\left(F_{n}\right)$ of finite subsets of $M$, where

$$
F_{n}=\left\{\lambda\left(T_{j}\right): j \leqslant n\right\} \cup\left\{\rho\left(T_{j}\right): j \leqslant n\right\}
$$

and

$$
E_{n}=\left(\left\{\lambda\left(T_{j}\right): j \leqslant n\right\} \cap M^{+}\right) \cup\left(\left\{\rho\left(T_{j}\right): j \leqslant n\right\} \cap M^{-}\right) .
$$

The intervals $T_{n}$ will have the following two properties.

(I) If $j<n$ and $T_{n} \cap T_{j} \neq \varnothing$, then $T_{n} \varsubsetneqq T_{j}$.

(II) If $j<n$ then $\pi_{j} \subset T_{n}$ only if $\lambda\left(T_{n}\right)=\inf \pi_{j}$ or $\rho\left(T_{n}\right)=\sup \pi_{j}$.

Let $E_{0}$ and $F_{0}$ be empty, and let $T_{0}=(-\infty, \infty)$. To proceed with the inductive step, assume that for all $j<k, T_{j}, E_{j}, F_{j}$ have been defined satisfying (I) and (II). Let $i_{k}=\min \left\{j: m_{j} \in M-E_{k-1}\right\}$. Then $m_{i_{k}}$ is an extreme point of $X \cap\left(-\infty, m_{i_{k}}\right)$ 
or of $X \cap\left(m_{i_{k}},+\infty\right)$ but not both. Assume the latter, then there are two cases.

(1) If $\left\{m \in M: m_{i_{k}}<m\right\}$ is empty (i.e., $m_{i_{k}}$ is the largest missing point of $D$ ), we put $E_{k}=E_{k-1} \cup\left\{m_{i_{k}}\right\}, F_{k}=F_{k-1} \cup\left\{m_{i_{k}}\right\}$ and $T_{k}=\left(m_{i_{k}},+\infty\right)$.

(2) If $\left\{m \in M: m_{i_{k}}<m\right\}$ is nonempty, then we define $W$ by

$W=\left\{m \in M: m_{i_{k}}<m\right.$ and $\mid\left\{\pi_{j}: j<k\right.$ and $\left.\pi_{j} \subset\left(m_{i_{k}}, m\right)\right\} \mid$ is minimum possible $\}$.

We note that if $m \in W$ then $\left(m_{i_{k}}, m\right)$ does not contain any $\pi_{j}$ with $j<k$ unless inf $\pi_{j^{\prime}}=m_{i_{k}}$ for some $j^{\prime}<k$. There are now two subcases.

(2a) If $W \cap F_{k-1}$ is nonempty, then put $m_{i_{k}}^{\prime}=\inf \left(W \cap F_{k-1}\right)$.

(2b) If $W \cap F_{k-1}$ is void, put $m_{i_{k}}^{\prime}=m_{j}$ where $j=\min \left\{r: m_{r} \in W\right\}$.

In both subcases, put $T_{k}=\left(m_{i_{k}}, m_{i_{k}}^{\prime}\right) ; F_{k}=F_{k-1} \cup\left\{m_{i_{k}}, m_{i_{k}}^{\prime}\right\}$ and $E_{k}=E_{k-1} \cup$ $\left\{m_{i_{k}}\right\}$ if $m_{i_{k}}^{\prime} \in M^{+}$or else $E_{k}=E_{k-1} \cup\left\{m_{i_{k}}, m_{i_{k}}^{\prime}\right\}$ if $m_{i_{k}}^{\prime} \in M^{-}$. We omit the routine proof that the inductive hypothesis is satisfied. We now define $\mathcal{S}$, a family of open intervals in $(D,<)$ by $\mathcal{S}=\left\{T_{k}: k \in \omega\right\}$. The family $\mathcal{S}$ has the following properties.

(1) For each $S \in \mathcal{S}, \lambda(S), \rho(S) \in M \cup\{-\infty,+\infty\}$.

(2) $\{\lambda(S): S \in \mathcal{S}\} \supset M^{+}$and $\{\rho(S): S \in \mathcal{S}\} \supset M^{-}$.

(3) For any $S, T \in \mathcal{S}$, either $S \cap T=\varnothing$ or $S \subsetneq T$ or $S \supsetneq T$.

(4) For every $S \in \mathcal{S}, \mathfrak{I}(S)=|\{T \in \mathcal{S}: T \supset S\}|$ is finite.

(5) If $S_{n} \in \mathcal{S}(n \in \omega)$, and $S_{n} \supset S_{n+1}$ for all $n$, then $\left|\cap S_{n}\right| \leqslant 1$.

Conditions (1) and (2) are clear. (3) and (4) amount to saying that $(\mathcal{S}, \supset)$ is a tree of height $\leqslant \omega$ and levels $\mathcal{S}_{n}=\{S \in \mathcal{S}: \mathfrak{l}(S)=n\}$; they follow from (I). Condition (5) follows from the definition of $W$, which implies that $\pi_{j}$ is contained in at most two elements $T_{k}$ of $\mathcal{S}$ for which $k>j$, and then only when $\lambda\left(T_{k}\right)$ or $\rho\left(T_{k}\right)$ is an extreme point of $\pi_{j}$.

We now define an order $<_{\omega}$ on $D$ as follows.

For $x, y \in D$ with $x<y$, let $n(x, y)=|\{S \in \mathcal{S}: x, y \in S\}|$; by conditions (3) to (5), $n(x, y)<\omega$. We put $x<_{\omega} y$ if $n(x, y)$ is even, $y<_{\omega} x$ otherwise. It is straightforward to check that $<_{\omega}$ is transitive, and hence a linear order on $D$. (It may be thought of as a limit of orderings: define $<_{0}$ by inverting $<$ on each member of $S_{0}(=\{D\})$, then define $<_{1}$ by inverting $<_{0}$ on each member of $S_{1}$, etc. Conditions (3) to (5) state that for any pair of distinct elements $x, y \in D$, there is $n_{0} \in \omega$ such that if $n \geqslant n_{0}$, then $x<_{n} y$ if and only if $x<_{n_{0}} y$. Thus the limit order is well defined.)

Let $t$ (resp. $t_{\omega}$ ) be the topology induced on $X$ by $<\mid X$ (resp. $\left.<_{\omega} \mid X\right)$, and let $t_{r}$ be the relative topology on $X$ that is inherited from $(D,<)$. We shall show that $t_{r}=t \vee t_{\omega}$.

We begin with two simple observations. First, $t_{r} \supset t$. Second, suppose that $x \in X$ is an element of infinitely many members of $\delta$; it is easy to see that these (or their traces on $X$ ) form a local base at $x$ in any of the spaces under consideration. ( $X$ is < -order dense in $X \cup M$; in particular, $X \cap S \neq \varnothing$ for each $S \in \mathcal{S}$.)

We now show that $t_{r} \subset t \vee t_{\omega}$. Fix $x \in X$ and suppose that $x$ is not a $t_{r}$-limit point of a set $A \subset X$. We assume that $x$ is a $t$-limit point and show that it not a 
$t_{\omega}$-limit of $A$. Thus we may assume, say, that $A \subset(x,+\infty)_{<}$(where $(,)_{<}$denotes an open interval in the order $<$ ), and so there is a missing point $x^{+}$. Let $k=\min \left\{j \in \omega: x^{+}=\lambda(S)\right.$ for some $\left.S \in \mathcal{S}_{j}\right\}$, and fix $S \in \mathcal{S}_{k}$ such that $x^{+}=$ $\lambda(S)$. Let $\mathcal{T}=\left\{T \in \mathcal{S}: T \subset S\right.$ and $\left.T \in \mathcal{S}_{k+1}\right\}$. There are now three cases to consider.

(a) If $\mathcal{T} \neq \varnothing$ and there exists $T \in \mathcal{T}$ such that $\lambda(T)>\lambda(S)$, then we can assume that $A \subset(x, \lambda(T))_{<}$and pick $y \in T$. It is easy to see that $y$ is $<_{\omega}$-between $x$ and A.

(b) If no $T$ as in (a) exists, but $\mathcal{T} \neq \varnothing$, then by (3) there is $T \in \mathcal{T}$ such that $\rho(T)<\rho(S)$. Thus we can fix $y \in S-T$ and assume that $A \subset(x, y)_{<}$. Again it is clear that $y$ is $<_{\omega}$-between $x$ and $A$.

(c) If $\mathcal{T}=\varnothing$, then we may fix $y \in S$ and assume that $A \subset(x, y)_{<}$. Then $y$ is $<_{\omega}$-between $x$ and $A$.

In all three cases it follows that $x$ is not a $t_{\omega}$-limit point of $A$ and hence that $t_{r} \subset t \vee t_{\omega}$.

To complete the proof we must show that $t_{r} \supset t_{\omega}$. To this end, fix $x \in X$, and suppose that $x$ is a $t_{r}$-limit point of a set $A \subset X$; we may further assume that $A \subset(x,+\infty)_{<}$, so that there is no missing point of the form $x^{+}$. By the second of the above observations, we may assume that there is a smallest $S_{x} \in \mathcal{\delta}$ containing $x$ (otherwise $x$ is clearly a $t_{\omega}$-limit point of $A$ ), and that $A \subset\left(x, \rho\left(S_{x}\right)\right)_{<}$. Now suppose that $n=\mathfrak{I}\left(S_{x}\right)+1$ and let $\mathcal{T}=\left\{T \in \mathcal{S}_{n}: T \subset\left(x, \rho\left(S_{x}\right)\right)_{<}\right\}$. If there is $y \in X$ such that $x<y<z$ for all $z \in A \cap \cup \mathcal{T}$ (which is vacuously true if $A \cap$ $\cup \mathcal{T}=\varnothing$ ), we may assume that $A \cap \cup \mathcal{T}=\varnothing$. Pick $a \in A$. Then we have

(i) $x$ is a $t_{r}$-limit point of $A \cap[x, a]_{<}$,

(ii) $<$ and $<_{\omega}$ agree up to inversion on $\left(A \cap[x, a]_{<}\right) \cup\{x\}$, and

(iii) $[x, a]_{<}$is order-convex in the order $<_{\omega}$, and so $x$ is a $t_{\omega}$-limit point of $A$.

If no such $y$ exists, then we may assume that $A \subset \cup \mathcal{T}$, and that $|A \cap T|<1$ for each $T \in \mathcal{T}$. Moreover, the orders induced on the quotient set $\left[x, \rho\left(S_{x}\right)\right)_{<} / \mathcal{T}$ (obtained by collapsing each element of $\mathcal{T}$ to a point) by $<$ and $<_{\omega}$ are identical up to inversion and so once again $x$ is a $t_{\omega}$-limit point of $A$. Hence $t_{r} \supset t_{\omega}$.

Thus $t_{r}=t \vee t_{\omega}$, as required.

\section{REFERENCES}

[C] E. Čch, Topological spaces, 2nd ed., Wiley, New York, 1966. MR 35 \#2254.

[DM] G. De Marco, A characterization of $C(X)$ for $X$ strongly paracompact, Sympos. Math. 21 (1977), 547-554. MR 57 \#7506.

[F] M. J. Faber, Metrizability in generalized ordered spaces, Math. Centre Tracts, no. 53, Mathematisch Centrum, Amsterdam, 1974. MR 54 \#6097.

[H] H. Herrlich, Ordnungsfahigkeit total-diskontinuierlicher Räume, Math. Ann. 159 (1965), 77-80.

[L 71] D. J. Lutzer, On generalized ordered spaces, Dissertationes Math., no. 89, PWN, Warsaw, 1971. MR 48 \#3018.

[L 80] __ Review of 'GO-spaces and generalizations of metrizability' by J. M. van Wouwe, Bull. Amer. Math. Soc. (N. S.) 3 (1980), 886-891.

[M 71] P. R. Meyer, On total orderings in topology, General Topology and its Relations to Modern Analysis and Algebra, III (Proc. Third Prague Topological Sympos., 1971), Academia, Prague, 1972, pp. 301-306. MR 52 \#9151.

[M 73] _ The Sorgenfrey topology is a join of orderable topologies, Czechoslovak Math. J. 23 (1973), 402-403. MR 47 \#7711. 
[P 77] S. Purisch, Orderability and suborderability of metrizable spaces, Trans. Amer. Math. Soc. 226 (1977), 59-76. MR 55 \# 1321.

[P 81] _ Scattered compactifications and the orderability of scattered spaces, Topology Appl. 12 (1981), 83-88.

[WM] R. G. Wilson and P. R. Meyer, Cardinal functions on products of orderable spaces (submitted).

Department of Mathematics, lehman College of the City University of New York, Bronx, NeW YORK 10468

Instituto de Matemáticas, Universidad Nacional Autónoma de México, México 20, D. F. México

Departamento de Matemáticas, Universidad Autónoma Metropolttana, Unidad Iztapalapa, México 13, D. F. México 